\title{
Strengthening Tuberculosis Prevention and Control in Nigeria: Exploring Integrated Approach
}

\author{
Aneke FA ${ }^{1}$, Chukwura DEI ${ }^{1}$, Ogbonna BO*2, Ajagu N ${ }^{2}$, Uzodinma SU², Epundu UU ${ }^{3}$, Onyeyili $\mathrm{A}^{4}$ and Earnest Orji CE ${ }^{5}$ \\ ${ }^{1}$ Department of Applied Microbiology, Nnamdi Azikiwe University, Awka, Nigeria
}

${ }^{2}$ Department of Clinical Pharmacy and Pharmacy Management, Nnamdi Azikiwe University, Nigeria

${ }^{3}$ Department of Community Medicine, Nnamdi Azikiwe University Teaching Hospital Nnewi, Nigeria

${ }^{4}$ Department of Nursing Services, Nnamdi Azikiwe University Teaching Hospital Nnewi, Nigeria

${ }^{5}$ Department of Pharmacology and Toxicology, Nnamdi Azikiwe University, Nigeria

*Corresponding author: Ogbonna BO, Department of Clinical Pharmacy and Pharmacy Management, Faculty of Pharmaceutical Sciences, Nnamdi Azikiwe University, Nigeria

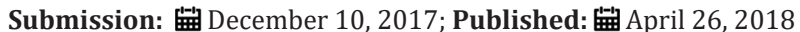

\begin{abstract}
Tuberculosis (TB) is an infectious disease caused by mycobacterium tuberculosis, and usually affects the lungs and other parts of the body. Patients with HIV infection are particularly more susceptible to wider varieties of mycobacterium strains. This study discussed integrated strategies of tuberculosis prevention and control for improved population health. The study discussed relevant documents and literatures written in English Language from reorganized websites. Key words were used individually and in series with truncations where necessary.

Government can aid the control of tuberculosis by providing good shelter, improved mass literacy programmes on the predisposing factors and prevention. The need to improve the standard of living, reduction of hunger and starvation is essential. The incidence worldwide stood at the rate of 10-20/100,000 per year with low mortality; deaths occurring mainly in the elderly and occasionally in undiagnosed cases. About $10 \%$ of all HIV-positive individuals harbor TB. That is 400 times the rate associated with the public. Only 3-4\% of infected individuals develop the active disease upon initial infection, $5-10 \%$ within one year. In cases of HIV infection the percentages are much higher because HIV infection tends to fuel the reactivation of existing TB infection as well as promote new infections. Integrated strategies will reduce the prevalence of TB especially among the HIV positive patients that are more vulnerable. Promotion of rational drug use, scaling up disease surveillance, monitoring and evaluation, and improving access to diagnostic centers are essential for improved prevention and control. Government policies targeted towards overcrowding prevention, and increased funding for TB research and development will boost and sustain already existing efforts. These processes in a continuum will boost sustainability.
\end{abstract}

Keyword: Tuberculosis; Drug resistance; Disease prevention; Public health; Health promotion; Nigeria; Patient care; Rational drug use

\section{Introduction}

Tuberculosis is an infection of the lower respiratory tract caused by the acid-fast bacilli Mycobacterium tuberculosis. It was first isolated in 1982 by a German physician named Robert Koch [1]. Mycobacterium tuberculosis is a rod-shaped, aerobic and nonspore forming bacteria. The most frequent mode of transmission is by droplet infection [2]. Close contact (e.g. in medical personnel) makes transmission by droplet nuclei most likely. The milk of tuberculosis cow is a source of infection where bovine tuberculosis is not well controlled and where milk is not pasteurized. Since the infection is mainly by inhalation of droplets nuclei suspended in the air, there is the possibility of one individual transmitting the infection to numerous healthy people. Therefore, there is the need to control the spread of the infection by prevention and control, case detection and rational treatment.
Certain measures can control the spread of TB. These include prevention of transmission at international borders through strict surveillance. Individuals have double responsibility to avoid either being infected or transmitting the infection to others [3]. In the case of already existing infection, patients movement have to be restricted to reduce social contacts to a minimum. The slogan, "Cough and sneezes spread diseases; trap the germs in your handkerchief" should be promoted and upheld through mass mobilization. Spitting out sputum on to the ground, where it dries easily, is a dangerous practice because the organism might be aerosolized [4]. Raising host resistance is essential to control or prevention of the infection. Malnutrition, long and rational use of antibiotics reduces resistance development. There is need for accelerated and improved immunization. Bacillus Calmette Guerin (BCG) vaccine 
has been found to be very effective against tuberculosis [5]. This study discussed integrated strategies of tuberculosis prevention and control for improved population health.

\section{Discussion}

\section{Dynamics of TB infection}

Previously, tuberculosis was considered to be eradicated in the developing countries due to the introduction of BCG and drug (Isonizid, Rifampicin, Pyrazinamide, etc) which was administered against tuberculosis and found to be effective [6]. Tuberculosis has resurfaced due to the emergence of antibiotic resistant strains of Mycobacterium tuberculosis called multi drug resistant tuberculosis (MDR- TB) or extreme drug resistant tuberculosis (XDR) [7]. Tuberculosis is a disease of global importance (A global emergency [8]. One-third of the world population is infected with Mycobacterium tuberculosis and eight million new cases of tuberculosis arise each year worldwide. The tuberculosis crisis is escalating since the human immunodeficiency virus (HIV) epidemic has triggered an even greater increase in the number of tuberculosis cases $[9,10]$. The majority of tuberculosis patients are 15 to 45 years of age, persons in their most productive years of life [11].

Tuberculosis kills over two million people worldwide each year more than any other single infectious disease, including AIDS and Malaria. Every year, more than 1.5 million TB cases occur in sub Saharan African [12]. Nearly 3 million cases occur in South - East Africa; 3 million cases of Acquired Immune Deficiency Syndrome (AIDS) with co-existent mycobacterium infection [13]. This increased tuberculosis prevalence threatens health services in Nigeria. Numerous factors account for this resurgence of TB and include; emergence of multi drug resistant strains of M. tuberculosis, erosion of accredited systems for diagnosis and standard treatment of the disease(s); immigration of infected persons from other countries where tuberculosis is prevalent [14]. About 5,000 and 10,000 tubercle bacilli per millilitre of sputum are required for direct microscopy to be positive and only a proportion of tuberculosis patient, harbor large enough numbers of organism to be detected in this way.

It is virtually impossible to distinguish different mycobacterium species by microscopy. Patients who have positive smears carry the greatest number of tubercle bacilli, and are the most infectious and are therefore the most important patients to detect early because they are responsible for spreading tuberculosis diseases. Sputum examination by microscopy is relatively quick, easy, and inexpensive and performed on cases suspected of having tuberculosis. Smear microscopy is used to monitor treatment progress and control programme outcome. Examination by bacteriological culture provides the definitive diagnosis of tuberculosis. Depending on the decontamination method and the type of culture medium used, as few as ten viable tubercle bacilli could be detected. However, the usual microbiological techniques of plating clinical material on selective or differential culture media and sub-culturing to obtain pure culture cannot be applied to tuberculosis bacteriology.
Compared to other bacteria, which typically reproduce within minutes, M. tuberculosis proliferates extremely slowly with a generation time of 18-24 hours [15-17].

The only media, which allow abundant growth of M.tuberculosis, are egg-enriched media containing glycerol and asparagines, and agar or liquid medium supplemented with serum or bovine albumin [18]. Culture increases the number of tuberculosis cases found, often by $30-50 \%$, and detects cases earlier, before they become infectious. Since culture techniques can detect few bacilli, the efficiency of diagnosing failures at the end of treatment could be improved. Culture of specimens is however, much more costly than microscopy and requires facilities for media preparation as well as skilled labour. Culture should be used selectively, in the following order of priority: surveillance of tuberculosis drug resistance as an integral part of the evaluation of control programme performance. Diagnosis of cases with clinical and radiological signs of pulmonary tuberculosis where smears are repeatedly negative; Diagnosis of extra-pulmonary and childhood tuberculosis; follow-up of tuberculosis cases who fail a standardized course of treatment and why many may have the risk of harboring drug-resistant organisms; should be upheld. These procedures should be employed in the investigation of high-risk individuals who are symptomatic, e.g. Laboratory workers, health care workers looking after multidrug resistant patients.

\section{Tuberculosis in Africa}

In the 1980s, the number of reported cases of TB in African increased dramatically. This increase was, however known to be minimal since most developing countries case notification amounts to only a fraction of the incidence. More than $95 \%$ of new TB case and tuberculosis-related deaths occur in individuals living in developing countries, predominantly in Asia and Africa with an estimated 5.4 million new cases in 1990. Sub-Saharan Africa is the region with highest incidence of tuberculosis worldwide. Globally there are 9.4 million persons with HIV and tuberculosis co-infection, of which the great majority were in sub-Sahara Africa (6.6 million). In 1993, the estimated proportion of TB cases attributable to HIV infection was $9.0 \%$ globally but $6 \%$ in sub-Sahara Africa. The increase in the TB in sub-Sahara Africa may have resulted from several factors including civil conflict leading to displacement, overcrowding, famine, and malnutrition. Together with economic decline these have in many cases led to a breakdown in health infrastructure. Reduced case finding and poor contact tracing led to an increase in the number of chronic tuberculosis excreters and careers [19-23].

\section{Control of Multi Drug Resistance- TB (MDR- TB)}

Considering the fact that the basis for admitting cases in the clinic is not based on whether patients are sputum positive (open case) or negative (closed case), but how 'severely debilitated' the patient is. It implies that open cases may be allowed to go away receiving treatment as an out-patient thus spreading the disease to individuals either in close proximity with them or through sputum from the patients which may be carried and blown away by wind etc. Contact tracing should be employed and suspects 
screened. Those infected can be treated under close supervision especially considering the fact that re-treatment cases are up to about $40 \%$ with the aim of avoiding further spread and the issue of passing resistant strains to the populace thus compounding the problems. Individuals, who came from the neighboring countries where there is outbreak of MDR-TB in one of their cities, and where nosocomial transmission was observed within their hospital, using the immigration records or patient's bio-data especially country of residence or travel history, should be traced. Individuals that had contact with the visitors of that country with the MDR-Tb outbreak should be traced to contain the spread of the MDR-TB.

In view of the fact that there is complex interaction between HIV and TB, patients with HIV have higher chances of progressing to active TB disease either from a recent or latent infection thus altering the course of TB in these individuals. The cases of MDR-TB in HIV infected patients is reduced by improved infection control measures on the wards and ensuring that patients complete their complete course of anti-TB treatment using the standard regimen of six drugs [24-27].

TB cases, whether new or re-treatment, should be treated aggressively especially within the first two months of the intensive phase to prevent the emergence of MDR-TB and to achieving total cure. This could be achieved preferably on an in-patient or under direct supervision in compliance with the objectives of 'DOTS' strategy. The new cases should be treated with an initial (intensive) phase of two months using four drugs (rifampicin (R), isoniazid (H), ethambutol (E), pyrazinamide $(\mathrm{Z})$ and a continuation phase lasting four to six months (using H/E) in accordance with the guidelines observed at the clinic as well. Re-treatment cases (patients who despite being treated for more than a month are still smear or culture positive), have higher chances of developing MDR-TB.

They should be treated in accordance with the treatment guidelines of the clinic (i.e. two months of streptomycin (S), R, H, E, Z, a month of $E / H / R / Z$, and five months of $H / R / E$ ). It should be mentioned that re-treatment of $\mathrm{TB}$, which often arise due to variety of factors such as patient abandonment or poor adherence to treatment, drug failure, microbial relapse etc, is one of the most difficult aspect of the management of this disease. This is so due to lack of personnel in this regards and or coupled with the fact that if second line drugs (amikacin, capreomycin, cycloserine, ethionamide, flurorquuinolones, kanamycin, p-amino salicylic acid, and thiacetazone) are used, treatment will be expensive. As a rule of thumb, a single drug should not be added to a failing regimen to avoid treatment failure and resistance [28-31].

In view of the fact that international donors enhance capacity for TB control, the following should be observed: Detailed history of drugs used by the patient should be taken so that when placing the patient on treatment. The drugs that should be used for treatment should be the ones, which the patient has never used before. Routine culture based drug sensitivity testing should be observed in accordance with international quality control standards since there is a national reference laboratory to facilitate this. The aim of this is to be able to select at least two, or preferably three new drugs based on the sensitivity testing and which the patient has never used before. Thus second-line drugs should be given based on appropriate drug susceptibility test, which requires standard laboratory facilities [32]. Re-treatment schemes should be managed by a specialist in that field with the view to prevent an outbreak of MDR- tuberculosis. On establishing that the patient is having tuberculosis, susceptibility testing should be performed with every initial isolate in order to assess drug resistance among new cases and to treat the patient in accordance with these results.

Early detection of MDR- tuberculosis can enhance early planning and further improve treatment strategies. There is often a significant delay in starting treatment in MDR- tuberculosis patients because of the fact that conventional susceptibility testing takes a period of at least three to four weeks. Although faster methods for rapid drug sensitivity testing are not yet widely established, studies have shown some promising approaches to that. In view of the fact that rifampicin is the most efficient of the anti-TB cocktail and coupled with the fact that MDR-TB depends on resistance to either rifampicin or isoniazid, rapid sensitivity testing for rifampicin will be of great advantage . To ensure patient adherence and avoid irrational prescription, DOTS strategy must be observed, in the case of MDR- tuberculosis, treatment should not be for duration of two years despite the fact that there are serious unwanted effects associated with the drugs [33-36].

\section{Public Health Measures in TB Prevention and Control}

Poverty reduction or elimination, good nutrition, improving socio-economic condition of patients, good housing to prevent overcrowding and ensure free air circulating with natural solar UV exposure, good occupational health policy in various working places, and support from both government and NGOs (nongovernmental organizations) towards expansion and application of the directly observed treatment short course (DOTS) strategy. To achieve these and many more, there must be political commitment on the side of government to support and sustain the backing of international donor agencies towards sustainable tuberculosis control. The strength of the personnel in the clinic should be improved, because lack of manpower would lead to non-adherence to the hospital treatment policies and failure to the DOTS strategies $[37,38]$. Specialist training and staff motivation should be given priority as well. In addition to the standardized supervised short course treatment, drugs should be administered on an outpatient basis preferably and under observation, as frequent hospitalization will deplete the already meagre resources.

However, high-risk cases (smear positive) should receive treatment as in-patients until when they become smear negative, with a view to curtailing spread of the infection. Vaccination using BCG, though has a limited role in the prevention of tuberculosis with practically zero epidemiological advantage since it does not act on the sources of infection, would go a long way in reducing serious tuberculosis presentations especially in children and also in preventing breakdown of the primary complex from causing milliary

How to cite this article: Aneke F, Chukwura D, Ogbonna B, Ajagu N, Uzodinma S, et al. Strengthening Tuberculosis Prevention and Control in Nigeria: 
or tuberculosis meningitis. Chemoprophylaxis using especially isoniazid, though of limited epidemiological impact as it does not act on the sources of infection, is getting popular in HIV patients, children and other infected individuals at risk of developing active tuberculosis. Use of mask and oral protectors are equally important measures to adopt in a hospital setting both the MDR-tuberculosis patients and health personnel in close contact with them with a view to preventing cross infection to other patients and risking the health personnel to the disease [37-40].

This study underscores the importance of strengthening the vast number of tuberculosis control measures both in the community and hospital environment with a view to reducing the high number of cases of TB seen among HIV patients, a common co-morbid condition seen in those who die of AIDS. Although HAART protects against tuberculosis by restoring patients' immune competence, TB screening, chemoprophylaxis with INH are essential in HIV positive patients and strict adherence to DOTS are of absolute importance in diagnosed with the infection. This would also go a long way in preventing the emergence of MDR- tuberculosis and XDR- tuberculosis, which has major implications for the care of HIV patients and the community in general. Tuberculosis is a disease of global importance. One-third of the world population was estimated to be infected with TB and eight million new cases of tuberculosis arise each year worldwide. The tuberculosis crisis is escalating since the human immunodeficiency virus (HIV) epidemic has triggered an even greater increase in the number of tuberculosis cases. The majority of tuberculosis patients are 15 to 45 years of age, persons in their most productive years of life $[9,11]$.

This is true about HIV/AIDS patients. Tuberculosis kills over two million people worldwide each year; more than any other single infectious disease, including AIDS and Malaria. According to W.H.O, every year, more than 1.5 million TB cases occur in sub Saharan African. Nearly 3 million cases occur in South - East Africa; Nearly 3 million cases occur in Acquired Immune Deficiency Syndrome (AIDS) with co-existent, mycobacterium infection This bring back tuberculosis in Western cities and seriously threatens health services in Nigeria. Numerous factors account for this resurgence of Tuberculosis: they include; emergence of multi drug resistant strains of M. tuberculosis, erosion of accredited systems for diagnosis and treatment of the disease(s); immigration of infected persons from countries where tuberculosis is prevalent;Prevalence of human immune deficiency virus (HIV) infection among others. Transmission of tuberculosis is virtually by droplet nuclei created through coughing by untreated persons suffering from pulmonary tuberculosis, (the most common forms) in a confined environment. Infected droplets remain airborne for a considerable time, and may be inhaled by susceptible persons [41,42]. Hospitalized care where patients are isolated in specialized wards is associated with psychosocial limitations that affect patients adversely [43]. A study in South Africa shows that decentralization through reduced hospitalization reduced cost of MDR-TB treatment [44]. There is a dire need to involve all stake holders to revive the End TB Strategy
2016-2035 for high burden countries (HBC) of which Nigeria are inclusive.

\section{Conclusion}

This work underscores the importance of strengthening the vast number of tuberculosis control measures both in the community and hospital environment with a view to reducing the high number of cases of TB seen in HIV patients, a common co-morbid condition seen in those who die of AIDS. Although HAART protects against tuberculosis by restoring patients' immune competence, TB screening, chemoprophylaxis with INH are essential in HIV positive patients and strict adherence to DOTS are of absolute importance in diagnosed with the infection. It will help in preventing increase in emergence of MDR- tuberculosis and XDR- tuberculosis, which has major implications for the care of HIV patients and the community in general. Tuberculosis is one of the most important infectious disease of the world and one that has made its impact felt through the ages. The burden of TB increased globally with the increase in HIV positive patients. Although the breakdown in the immune system do not often allow the HIV/AIDS patients to produce quality sputum wherein the detectable number of bacilli are seen. The use of cultural technique enhances the case detection rate. The turnaround time for result production in acid-fast bacilli (AFB; Zeihl Nelson) stain aids investigation. Preventive therapies should be prompt for prevention of TB progression to clinically active disease. Effective strategies fail due to lack of sustainability. However, integrating and sustaining these strategies in a continuum will reverse the trend.

\section{References}

1. Kumar V, Abbas AK, Fausto N, Mitchell N, Richard N (2007) Robbins Basic Pathology ( $8^{\text {th }}$ edn), Saunders Elsevier, pp. 516-522.

2. Enarson DA, Murray JF (1996) Global epidemiology of tuberculosis: In Tuberculosis (Rom W N and Garay S) Little, Brown and Company, USA, pp. $57-75$

3. Hopewell PC, Pai M, Maher D, Uplekar M, Raviglione MC (2006) International standards for tuberculosis care. Lancet Infect Dis 6(11): 710-725.

4. World Health Organization (2004) TB/HIV A clinical manual $\left(2^{\text {nd }}\right.$ edn), Publication No WHO/HTM/TB/2004.329. World Health Organization, Geneva, Switzerland.

5. Crofton J, Chaulet P, Maher D (1997) Guidelines for the management of drug-resistant tuberculosis. Publication No. WHO/TB 96.210 (Rev. 1) World Health Organization, Geneva, Switzerland.

6. Bonah C (2005) The experimental stable of the BCG vaccine: safety, efficacy, proof, and standards, 1921-1933. Stud Hist Philos Biol Biomed Sci 36(4): 696-721

7. Center for Disease Control (2006) Emergence of Mycobacterium tuberculosis with Extensive Resistance to Second-Line Drugs Worldwide, 2000-2004. MMWR Morb Mortal Wkly Rep 55(11): 301-305.

8. Stead WW, Bates JH (1996) Geographic and evolutionary epidemiology of tuberculosis. In Tuberculosis (Rom WN and Garay S). Little, Brown and Company, USA. pp. 77-83.

9. World Health Organization (2009) Epidemiology Global tuberculosis control: epidemiology, strategy, finances. pp. 6-33. 
10. Gandhi NR, Moll A, Sturm AW, Pawinski R, Govender T, et al. (2006) Extensively drug-resistant tuberculosis as a cause of death in patients co-infected with tuberculosis and HIV in a rural area of South Africa. Lancet 368(9547): 1575-1580.

11. Extensively drug-resistant tuberculosis (XDR-TB) (2006): Recommendations for prevention and control. Wkly Epidemiol Rec 81(45): 430-432.

12. World Health Organization (2004) TB/HIV A clinical manual ( $2^{\text {nd }}$ edn) Publication No. WHO/HTM/TB/2004.329. World Health Organization, Geneva, Switzerland.

13. Chaisson RE, Martinson NA (2008) Tuberculosis in Africa-combating an HIV-driven crisis. N Engl J Med 358(11): 1089-1092.

14. World Health Organization (2003) Treatment of Tuberculosis: Guidelines for National Programmers ( $3^{\text {rd }}$ edn), Publication No. WHO/ CDS/TB/2003.313. World Health Organization, Geneva, Switzerland.

15. World Health Organization (2004) TB/HIV A clinical manual $\left(2^{\text {nd }}\right.$ edn) Publication No. WHO/HTM/TB/2004.329. World Health Organization, Geneva, Switzerland.

16. Nester EW, Anderson DG, Roberts CE, Nancy J, Pearsall NN, et al. (2004) Microbiology: A Human Perspective (4). McGraw-Hill, New York, USA.

17. Jawetz R, Melnick JK, Adelberg A (1997) Medical Microbiology (21 edn), pp. 278-289.

18. WHO (1998) World Health Organization laboratory services in Tuberculosis controls part II: microscopy: WHO/TB/98. 258. Geneva, Switzerland.

19. Federal Ministry of Health (FMH) (2000) TB and Leprosy control effects in Nigeria National TB \& Leprosy control Programme (NTBLCP).

20. WHO (2005) Global Tuberculosis Control, Surveillance, Planning Financing; WHO Report/HTM/TB/2005.349. Geneva, Switzerland.

21. World Health Organization (2014) Global tuberculosis report. Geneva, Switzerland.

22. WHO (2012) WHO policy on collaborative TB/HIV activities: guidelines for national programmes and other stakeholders. WHO Press, Geneva, Switzerland.

23. World Health Organization (2007) Report on global tuberculosis control: surveillance, planning, finances. World Health Organization, Geneva, Switzerland.

24. (2006) Emergency of Mycobacterium tuberculosis with extensive resistance to second-line drugs worldwide 2002-2004. MMWR Morb Mortal Wkly Rep 55: 301-305.

25. Gandhi NR, Moll A, Sturm AW, Pawinski R, Govender T, et al. (2006) Extensively drug-resistant tuberculosis as a cause of death in patients co-infected with tuberculosis and HIV in a rural area of South Africa. Lancet 368(9547): 1575-1580.

26. Zignol M, Hosseini MS, Wright A, Weezenbeek CL, Nunn P, Watt CJ, et al. (2006) Global Incidence of Multidrug-Resistant Tuberculosis. Journal of Infectious Diseases 194(4): 479-485.

27. Crofton J, Chaulet P, Maher D (1997) Guidelines for the management of drug-resistant tuberculosis. World Health Organization, Geneva, Switzerland.
28. Caminero JA (2004) Tuberculosis Guide for Specialist Physicians Paris: International Union against Tuberculosis and Lung Disease, pp. 228267.

29. World Health Organization (2003) Treatment of Tuberculosis: Guidelines for National Programmes ( $3^{\text {rd }}$ edn), World Health Organization, Geneva, Switzerland.

30. World Health Organization (2004) TB/HIV A clinical manual $2^{\text {nd }}(e d n)$, World Health Organization, Geneva, Switzerland.

31. World Health Organization (2000) Guidelines for Establishing DOTSPlus Pilot Projects. World Health Organization, Geneva, Switzerland.

32. Hannan M, Peres H, Maltez F, Hayward AC, Machado J, et al. (2001) Investigation and control of a large outbreak of multi-drug resistant tuberculosis at a central Lisbon hospital. J Hosp Infect 47(2): 91-97.

33. World Health Organization (2000) Guidelines for Establishing DOTSPlus Pilot Projects. World Health Organization, Geneva, Switzerland.

34. Roth A, Schaberg T, Mauch H (1997) Molecular diagnosis of tuberculosis: current clinical validity and future perspectives. Eur Respir J 10: 18771891.

35. Heifets LB, Cangelosi GA (1999) Drug susceptibility testing of Mycobacterium tuberculosis: a neglected problem at the turn of the century. Int. J Tuberc Lung Dis 3(7): 564-581.

36. Adegboyega TT, Thomas BT, Agu GC, Abiodun AT 2014Can Nigeria Sustain the Fight against Drug Resistant Mycobacterium tuberculosis? Journal of Microbiology Research 4(2): 72-77.

37. World Health Organization (2009) Epidemiology. Global tuberculosis control: epidemiology, strategy, finances. pp. 6-33.

38. Dye C, Watt CJ, Bleed DM, Williams BG (2003) what is the limit to case detection under the DOTS strategy for tuberculosis control? Tuberculosis (Edinb) 83(1-3): 35-43.

39. Comstock G (1994) The International Tuberculosis Campaign: a pioneering venture in mass vaccination and research. Clin Infect Dis 19(3): 528-540.

40. World Health Organization (2007) Report on global tuberculosis control: surveillance, planning, financing. World Health Organization, Geneva, Switzerland.

41. World Health Organization (2015) What is multidrug-resistant tuberculosis and how do we control it?

42. Lezor BK, Weigel R, Smith H (2017) Hospitalized care for MDR-TB in Port Harcourt, Nigeria: a qualitative study. BMC Infect Dis 17(1): 50.

43. Sinanovic E, Ramma L, Vassall A, Azevedo V, Wilkinson L, et al. (2015) Impact of reduced hospitalisation on the cost of treatment for drugresistant tuberculosis in South Africa. Int J Tuberc Lung Dis 19(2): 172178.

44. WHO (2018) High TB burden country lists 2016-2020 - background paper. 
Creative Commons Attribution 4.0 International License

For possible submissions Click Here

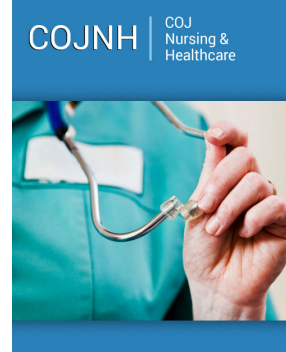

\section{COJ Nursing \& Healthcare}

\section{Benefits of Publishing with us}

- High-level peer review and editorial services

- Freely accessible online immediately upon publication

- Authors retain the copyright to their work

- Licensing it under a Creative Commons license

- Visibility through different online platforms 